\section{Case Reports in Dermatology}

\title{
Discrete Papular Lichen Myxedematosus and Scleromyxedema with Hypothyroidism: A Report of Two Cases
}

\author{
Adele Shenoy $^{a} \quad$ Jacklyn Steixner $^{b} \quad$ Vincent Beltrani $^{c} \quad$ Alice Gottlieb $^{d}$ \\ aSchool of Medicine, New York Medical College, Valhalla, NY, USA; bHudson Dermatology, \\ Somers, NY, USA; 'C Caremount Medical, Poughkeepsie, NY, USA; ${ }^{d}$ Department of \\ Dermatology, Icahn School of Medicine at Mount Sinai, New York, NY, USA
}

\section{Keywords}

Papular mucinosis · Lichen myxedematosus · Scleromyxedema

\begin{abstract}
Scleromyxedema and lichen myxedematosus (LM) are rare disorders that fall along the spectrum of primary cutaneous mucinoses. Scleromyxedema is a systemic form that classically presents with generalized waxy papules, sclerodermoid eruption, and monoclonal gammopathy; LM is a localized form limited to the skin that classically presents with white, firm, waxy papules and lacks monoclonal gammopathy. According to diagnostic criteria established in 2001, the diagnosis of both conditions requires absence of thyroid disease. However, atypical cases that lack monoclonal gammopathy and that present with hypothyroidism have been reported, suggesting that these criteria may require revision. First, we report a case of a 58-year-old female with a history of Hashimoto thyroiditis and biopsy-proven scleromyxedema responsive to intravenous immunoglobulin therapy with delayed presentation of monoclonal gammopathy. Next, we report a case of a 54-year-old female with a history of hypothyroidism, Hodgkin's lymphoma in remission after radiation and chemotherapy, and concurrent rheumatoid arthritis, with biopsy-proven LM temporarily responsive to systemic steroids. Our cases demonstrate that patients with papular mucinoses can have a multitude of concurrent and prior rheu-
\end{abstract}


matologic and endocrine conditions, including thyroid disease, which should not preclude a diagnosis of scleromyxedema and LM.

\section{Introduction}

Scleromyxedema and lichen myxedematosus (LM) are uncommon conditions characterized by cutaneous deposition of mucin. In 2001, Rongioletti and Rebora [1] proposed a set of diagnostic criteria for scleromyxedema that included generalized waxy papules and sclerodermoid eruption, a histological triad including mucin deposition, fibrosis, and fibroblast proliferation, and laboratory testing showing monoclonal gammopathy and absence of thyroid disease. Scleromyxedema can also involve the cardiovascular, nervous, gastrointestinal, musculoskeletal, and respiratory systems. Their diagnostic criteria for LM included papular or nodular eruption, mucin deposition with variable fibroblast proliferation, and absence of monoclonal gammopathy and thyroid disease [1]. They described five subtypes of localized LM: discrete papular LM, acral persistent papular mucinosis, self-healing papular mucinosis with adult and juvenile subtypes, papular mucinosis of infancy, and a nodular form.

However, not all reported cases of papular mucinosis fit these diagnostic criteria. There are some reported cases of scleromyxedema without monoclonal gammopathy, LM with monoclonal gammopathy, and scleromyxedema with thyroid disease, which are considered atypical forms [1]. In 2017, Nofal et al. [2] proposed two new sets of diagnostic criteria to facilitate the diagnosis of these atypical forms. The first set includes invariable clinical and histological characteristics that are present in all cases of LM: firm, waxy papules that may coalesce, and diffuse dermal mucin deposition and fibroblast proliferation with or without fibrosis. The second set includes features that vary amongst cases, including monoclonal gammopathy, thyroid disorder, HIV, hepatitis C, peripheral eosinophilia, thymic carcinoma, and hepatocellular carcinoma [2]. They distinguish scleromyxedema from purely cutaneous forms of LM based on the presence of systemic symptoms.

Here, we report two cases of patients with a history of thyroid disease whose clinical and histopathological findings represent atypical forms of scleromyxedema and discrete papular LM, respectively. Our cases demonstrate the abundance of concurrent and prior rheumatologic and endocrine conditions that should not preclude diagnosis, but rather expand upon the diagnostic criteria.

\section{Case 1}

A 58-year-old Caucasian female with a past medical history of Hashimoto thyroiditis had been well until 2012 when she noticed redness and thickening of the skin on the face, progressively spreading to the trunk and extremities. The rash was neither painful nor pruritic. She reported no history of cardiovascular, neurological, nor respiratory symptoms, though she reported occasional joint pains. Her hypothyroidism had been well managed on levothyroxine. Skin biopsy in 2012 showed an increased amount of mucin deposition, increased fibroblasts, and a perivascular infiltrate of lymphocytes, histiocytes, and plasma cells. Laboratory testing in 2012 was significant for an elevated thyroid peroxidase antibody level of 1,113 IU/mL. Other labs including CBC, CMP, TSH, ANA, rheumatoid factor, ESR, CPK, anti-Ro, and anti-La were within normal limits. Serum protein electrophoresis was within normal limits. One year after diagnosis, in 2013, she was started on intravenous immunoglobulin therapy and 
responded well, with clearance of her skin after two infusions. However, once her dose was reduced in 2016, her dermatologic symptoms relapsed.

At current presentation in 2018, physical examination was significant for widespread indurated, waxy, erythematous, and thickened skin throughout the entire body. Erythematous, confluent papules measuring 2-4 mm were most visible on the face and chest (Fig. 1). Her back and thighs showed indurated skin folds consistent with the "Shar Pei sign" (Fig. 2). Bilateral forearms and legs lacked hair. Her fingers, upper extremities, and neck showed normal motility. Laboratory testing was significant for elevated IgG at 1,953 mg/dL (normal range $700-1,600 \mathrm{mg} / \mathrm{dL}$ ), and serum protein electrophoresis showed elevated gamma globulin at $2.1 \mathrm{~g} / \mathrm{dL}$ (normal range $0.4-1.8 \mathrm{~g} / \mathrm{dL}$ ), with a pattern demonstrating a single peak M-spike in the gamma region. Urinary analysis showed positive leukocyte esterase and 11-30 WBC/HPF associated with urinary tract infection, but normal immunofixation and electrophoresis.

\section{Case 2}

A 54-year-old Caucasian female with a past medical history of rheumatoid arthritis, hip replacement, Hodgkin's lymphoma treated with radiation and chemotherapy 25 years ago, and hypothyroidism presented after she had noticed an eruption of white bumps on her chest one year ago in 2017 that caused her distress. She reported that the waxy papules responded to courses of oral steroids, but always returned after stopping the therapy. Upon relapse, the papules appeared on her neck, chest, both axilla, forearms, and under the breasts. They responded to intramuscular steroids, but again relapsed. Methotrexate provided no resolution. At the time of diagnosis, her laboratory workup showed positive rheumatoid factor, but other labs including CBC, CMP, and ESR were within normal limits. Serum protein electrophoresis was within normal limits. Biopsy of a papule showed palisading lymphocytes and histiocytes around mucin. Her hypothyroidism was well managed with levothyroxine, but her rheumatoid arthritis still caused her joint pain despite trials of methotrexate and leflunomide.

At current presentation in 2018, physical examination showed asymptomatic, smooth, monomorphous, waxy, white 2-mm papules distributed on the chest, upper back, and left forearm (Fig. 3). There was no induration, edema, or erythema. Her rheumatoid arthritis was being treated with hydroxychloroquine. Repeat laboratory testing was significant for elevated rheumatoid factor at $32.0 \mathrm{IU} / \mathrm{mL}$ (normal range $<8.6-11.9$ ). Other labs including serum protein electrophoresis, $\mathrm{CBC}$, TSH, urinary analysis, and urine protein electrophoresis were within normal limits.

\section{Discussion}

The diagnostic criteria proposed by Rongioletti and Rebora in 2001 [1] would exclude a diagnosis of scleromyxedema and LM in our patients given their hypothyroidism. Our cases instead support the use of the updated diagnostic criteria suggested by Nofal et al. in 2017 [2], which consider monoclonal gammopathy and thyroid disease as varying rather than constant features.

Both of our cases demonstrate that a history of thyroid disease should not be an exclusionary criterion in the diagnosis of scleromyxedema and LM, especially if well managed on thyroid replacement therapy. Rongioletti and Rebora [1] may include the absence of thyroid disease as part of the diagnosis because hypothyroidism can present with generalized 
myxedema and histology showing mucin deposition [3]. However, there are multiple cases of scleromyxedema and LM in addition to ours that have been diagnosed with concurrent thyroid disease $[2,4,5]$. Our patients presented with forms of papular mucinosis years after hypothyroidism had been diagnosed and sufficiently treated. Although we cannot rule out an association between their hypothyroidism and mucin deposition, a causal relationship is unlikely in our cases.

It is important to distinguish hypothyroidism-induced mucinosis from scleromyxedema and LM due to potential differences in response to therapy. There are some cases of localized mucinosis associated with hypothyroidism that responded to thyroid replacement therapy [6, 7], but others that did not [8]. Sufficient thyroid replacement therapy in our patients did not improve their symptoms. However, scleromyxedema both in our patient and in others has shown response to its current first-line therapy, intravenous immunoglobulin, which often requires maintenance therapy to prevent relapse $[9,10]$. Discrete papular LM has shown response to topical steroids and topical tacrolimus, and systemic steroids provided temporary resolution in our patient $[1,11]$. Their demonstrated therapeutic response is further evidence that their mucinoses are likely unrelated to their well-managed thyroid disease.

In addition to hypothyroidism, our first case of scleromyxedema is unusual because she lacked monoclonal gammopathy at the time of her initial diagnosis. Though paraproteinemia is found in about $83 \%$ of patients, previous cases without gammopathy have been reported $[9,12]$. Our patient had monoclonal gammopathy years after diagnosis, while experiencing relapse of symptoms. Although her elevated serum IgG may be attributable to her most recent intravenous immunoglobulin dose received 7 days prior to laboratory work, the therapy would not be expected to cause monoclonal gammopathy. It is possible that this represents a delayed presentation of paraproteinemia associated with scleromyxedema, which has also previously been reported [1]. Thus, our case supports the inclusion of monoclonal gammopathy as part of the varying, rather than constant, features of scleromyxedema, as described by Nofal et al. [2].

Our second patient had characteristic findings of discrete papular LM including white, firm papules distributed throughout the body, and histology showing mucin deposition. This patient had an extensive past medical history including rheumatoid arthritis, hip replacement, Hodgkin's lymphoma, and radiation therapy. Interestingly, there is a prior report of a patient with rheumatoid arthritis and hip replacement presenting with self-healing papular mucinosis, a rare subtype of LM [13], as well as a report of papular mucinosis localized to the breast after radiation therapy [14]. Furthermore, it has been demonstrated that irradiated rheumatoid fibroblasts have an increased rate of hyaluronic acid production and that hydrocortisone inhibited this effect [15]. Thus, her rheumatoid arthritis and prior radiation therapy could be associated with her current increase in mucin deposition and its response to steroids. The association of rheumatoid arthritis, hip replacement, and radiation therapy with LM is limited to case reports, and establishing direct relationships between these conditions in our patient is difficult. However, these conditions may add to the variable features found in scleromyxedema and LM that are included in the second set of Nofal et al.'s [2] diagnostic criteria.

In conclusion, our cases support Nofal et al.'s [2] updated diagnostic criteria, which include thyroid disease and monoclonal gammopathy as varying features of scleromyxedema and LM. Our cases highlight the diversity of the papular mucinoses: our first case of scleromyxedema presented with delayed monoclonal gammopathy upon relapse of symptoms, and our second case presented with LM with concurrent rheumatoid arthritis and prior joint replacement and radiation therapy. Relationships between rheumatologic and endocrine conditions 
such as hypothyroidism and the papular mucinoses require further investigation so as to better establish diagnostic criteria and treatment strategies.

\section{Statement of Ethics}

Consent to publication of this report was obtained from both patients.

\section{Disclosure Statement}

The authors have no conflicts of interest to declare.

\section{Funding Sources}

The authors did not use any funding sources for this report.

\section{Author Contributions}

All authors participated in patient care, obtaining patient history and workup, and writing the manuscript.

\section{References}

1 Rongioletti F, Rebora A. Updated classification of papular mucinosis, lichen myxedematosus, and scleromyxedema. J Am Acad Dermatol. 2001 Feb;44(2):273-81.

2 Nofal A, Amer H, Alakad R, Nofal E, Desouky FE, Yosef A, et al. Lichen myxedematosus: diagnostic criteria, classification, and severity grading. Int J Dermatol. 2017 Mar;56(3):284-90.

3 Smith TJ, Bahn RS, Gorman CA. Connective tissue, glycosaminoglycans, and diseases of the thyroid. Endocr Rev. 1989 Aug;10(3):366-91.

4 Li L, Jiang C, Lu Y, Zhang P. A case of scleromyxoedema with thyroid dysfunction. Indian J Dermatol. 2014 Sep;59(5):524-5.

5 Volpato MB, Jaime TJ, Proença MP, Gripp AC, Alves MF. [Papular mucinosis associated with hypothyroidism]. An Bras Dermatol. 2010 Jan-Feb;85(1):89-92.

6 Jakubovic HR, Salama SS, Rosenthal D. Multiple cutaneous focal mucinoses with hypothyroidism. Ann Intern Med. 1982 Jan;96(1):56-8.

7 Martin-Ezquerra G, Sanchez-Regaña M, Massana-Gil J, Umbert-Millet P. Papular mucinosis associated with subclinical hypothyroidism: improvement with thyroxine therapy. J Eur Acad Dermatol Venereol. 2006 Nov;20(10):1340-1.

8 Ertam I, Karaca N, Ceylan C, Kazandi A, Alper S. Discrete papular dermal mucinosis with Hashimoto thyroiditis: a case report. Cutis. 2011 Mar;87(3):143-5.

9 Rongioletti F, Merlo G, Cinotti E, Fausti V, Cozzani E, Cribier B, et al. Scleromyxedema: a multicenter study of characteristics, comorbidities, course, and therapy in 30 patients. J Am Acad Dermatol. 2013 Jul;69(1):6672.

10 Blum M, Wigley FM, Hummers LK. Scleromyxedema: a case series highlighting long-term outcomes of treatment with intravenous immunoglobulin (IVIG). Medicine (Baltimore). 2008 Jan;87(1):10-20.

11 Rongioletti F, Zaccaria E, Cozzani E, Parodi A. Treatment of localized lichen myxedematosus of discrete type with tacrolimus ointment. J Am Acad Dermatol. 2008 Mar;58(3):530-2.

12 Dinneen AM, Dicken CH. Scleromyxedema. J Am Acad Dermatol. 1995 Jul;33(1):37-43.

13 Yokoyama E, Muto M. Adult variant of self-healing papular mucinosis in a patient with rheumatoid arthritis: predominant proliferation of dermal dendritic cells expressing CD34 or factor XIIIa in association with dermal deposition of mucin. J Dermatol. 2006 Jan;33(1):30-5. 


\section{Case Reports in Dermatology}

14 Fernandez-Flores A, Barja-Lopez JM, Saeb-Lima M. Papular mucinosis of the breast after radiation therapy. J Cutan Pathol. 2014 Dec;41(12):969-71.

15 Yaron M, Yaron I, Levita M, Herzberg M. Hyaluronic acid production by irradiated human synovial fibroblasts. Arthritis Rheum. 1977 Mar;20(2):702-8.

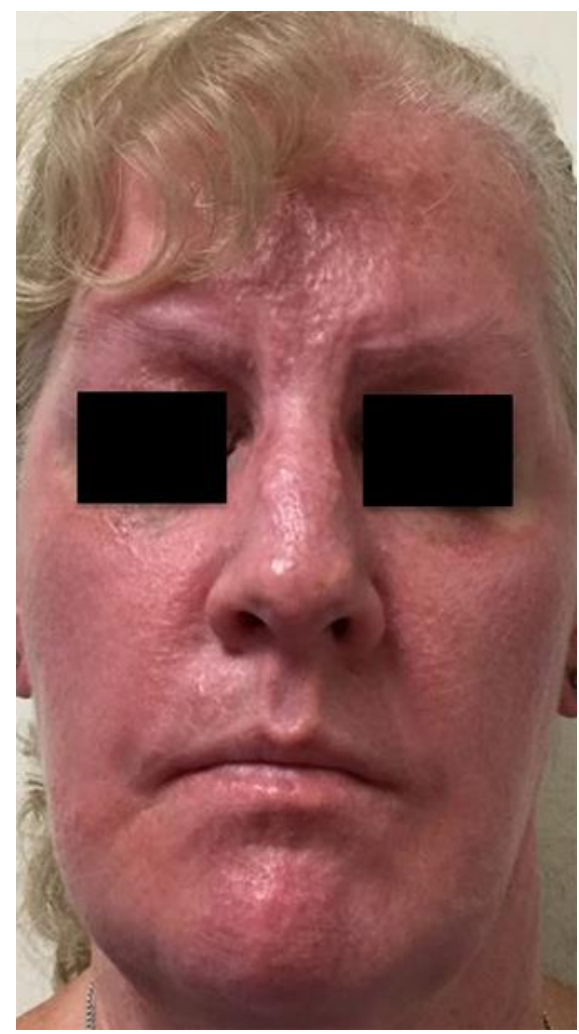

Fig. 1. Scleromyxedema with erythematous, confluent papules and waxy, thickened skin of the face. 


\section{Case Reports in Dermatology}
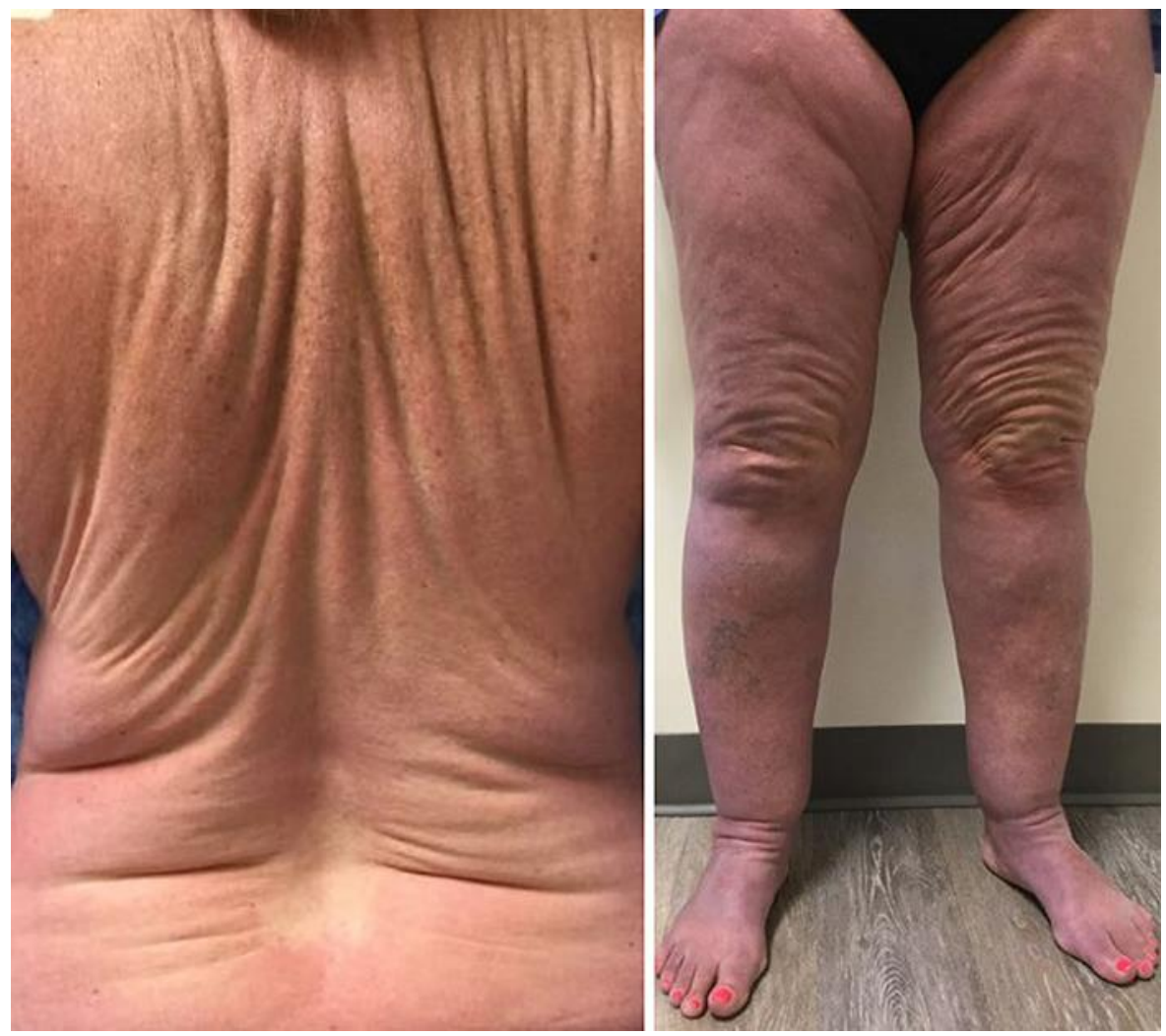

Fig. 2. Scleromyxedema with indurated, erythematous skin folds demonstrating the "Shar Pei sign" on the back (left) and bilateral legs (right).
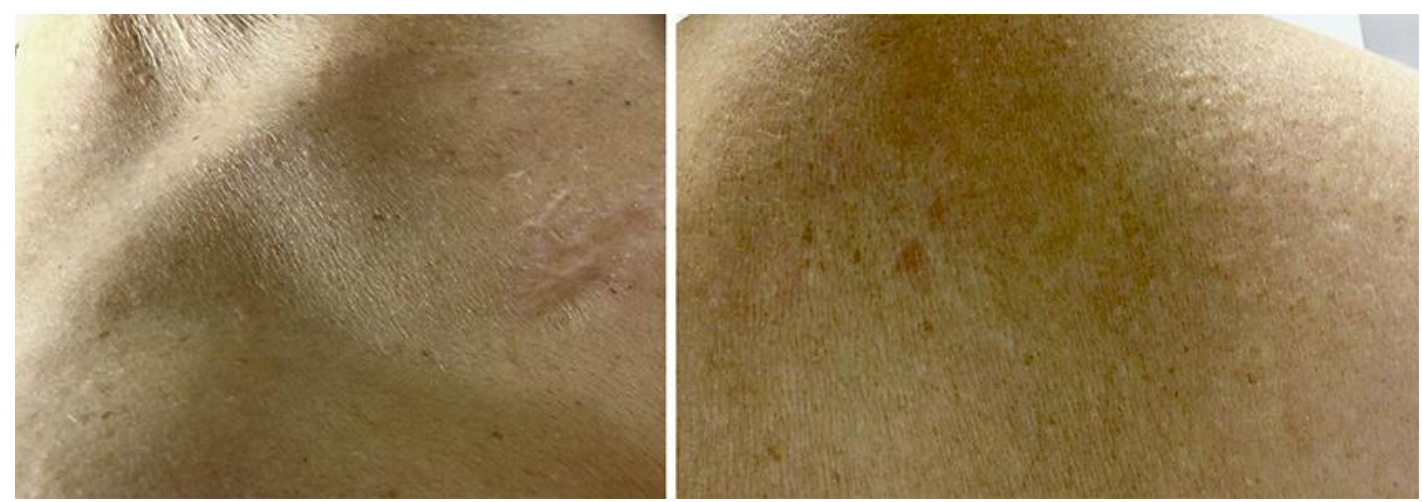

Fig. 3. Discrete papular lichen myxedematosus presenting with firm, smooth, 2-mm whitish to skin-colored papules on the neck (left) and upper back (right). 